\title{
The Jacobi constant for a cometary orbiter
}

\author{
E. Mysen and K. Aksnes
}

Institute of Theoretical Astrophysics, University of Oslo, PO Box 1029 Blindern, 0315 Oslo, Norway

e-mail: [eirik.mysen;kaare.aksnes] @astro.uio.no

Received 12 May 2005 / Accepted 29 July 2005

\section{ABSTRACT}

The Jacobi constant of a probe under the gravitational attraction of a rotating irregular body is rederived for excited, but free rigid rotation of the central mass. A related Tisserand-like quantity is found to be sufficiently conserved for it to qualify as a pseudo-integral. The quantity's near constancy is shown to imply that certain regions in the space of the probe's cometocentric orbital elements are forbidden. In particular, based solely on this analysis, it is seen how collision with the comet nucleus is best avoided if the initial probe velocity lies in the plane normal to the nucleus' rotational spin. A conclusion which is of relevance to the Rosetta mission where the lander Philae is to be delivered by Rosetta in a possibly close prograde, and therefore probably shape unstable, orbit. At the comet's heliocentric distance when the lander is delivered, radiation and radial outgassing pressure do not significantly affect these conclusions for a nominal target nucleus in a worst-case scenario. The latter effect is seen to somewhat decrease the impact risk of prograde trajectories, making the more stable retrograde orbits slightly less safe.

Key words. celestial mechanics - space vehicles - comets: general - comets: individual: 67P/Churyumov-Gerasimenko - methods: analytical - methods: numerical

\section{Introduction}

It is well known that close orbits around small irregular celestial bodies can experience gravitationally induced rapid, aperiodic variations in orbital energy. These changes occur at orbital distances and eccentricities for which the resonances between the nucleus spin and the orbiting probe's orbit have fused, forming a chaotic zone, no longer confined to the separatrices of the resonances, in which the actions of the system can wander (Lichtenberg \& Lieberman 1992). However, if the central body is rotating uniformly, there exists an integral, the Jacobi constant, constraining the probe's motion. Usually, these constraints are represented geometrically as socalled zero-velocity curves.

As witnessed for 1P/Halley by ESA's Giotto, the rotation of cometary nuclei could be excited. Therefore, in anticipation of cometary missions like Rosetta, the variation of the Jacobi function for a spacecraft in an orbit around a non-uniformly rotating central body will be studied. If a related function is found to be sufficiently conserved, the implications for any initial orbit will be derived.

Not untypical for short-periodic comets, the Rosetta target 67P/Churyumov-Gerasimenko is a relatively small (Lamy et al. 2003) low density object (Davidsson \& Gutiérrez 2005). Even though the isolated analysis of the gravitational problem places restrictions on the fast gravitationally induced changes of orbital energy, the effect of the possibly strong non-gravitational forces must therefore be taken into account if questions of orbital constraints on longer time-scales are to be raised.

\section{The nucleus}

The comet nucleus' gravitational field is modelled using a standard spherical harmonics expansion of the gravitational potential (Heiskanen \& Moritz 1967)

$$
\begin{aligned}
V_{1}= & \frac{\mu_{\mathrm{c}}}{r} \sum_{n=2}^{\infty} \sum_{m=0}^{n}\left(\frac{r_{\mathrm{c}}}{r}\right)^{n} P_{n m}(\sin \beta) \\
& \times\left[c_{n m} \cos m \lambda+s_{n m} \sin m \lambda\right] .
\end{aligned}
$$

Here $r$ is the distance between the probe and the mass center of the nucleus whose characteristic radius is $r_{\mathrm{c}}$, while $\lambda$ and $\beta$ are the spacecraft's east longitude and north latitude, respectively, in the nucleus' principal axis system (Goldstein 1980). $\mu_{\mathrm{c}}$ is the product of the constant of gravitation and the comet's mass, and $P_{n m}$ are the Legendre functions. The acceleration of an orbiting probe induced by gravitation is then

$$
\ddot{\boldsymbol{r}}=-\nabla\left(V_{0}+V_{1}\right), \quad \nabla=\sum_{i=1}^{3} \boldsymbol{u}_{X_{i}} \frac{\partial}{\partial X_{i}},
$$

with the Kepler potential $V_{0}=-\mu_{\mathrm{c}} / r$, where $X_{i}=X, Y, Z$ are the coordinates in the adopted cometocentric reference system, and $\boldsymbol{u}_{X_{i}}$ are unit vectors defining the reference axes. For convenience, the reference $X Y$-plane is assumed to coincide with the plane normal to the nucleus $\operatorname{spin} \boldsymbol{G}_{\mathrm{c}}$. 


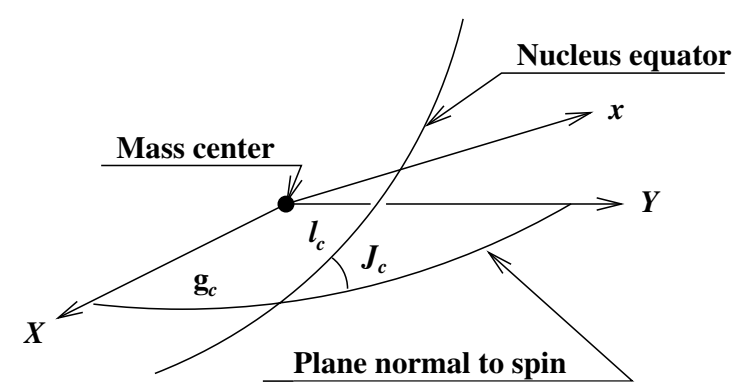

Fig. 1. The reference system, the principal $x$-axis and angles of rigid rotation.

In order to calculate the gradient Eq. (2), we have formulated $V_{1}$ in terms of the probe's Cartesian components $X_{i}$. As a first step, observe that each spherical harmonic easily (Heiskanen \& Moritz 1967) can be written as a function of the probe's Cartesian components $x_{i}=x, y, z$ in the nucleus' principal axis system, axes defined by the unit vectors $\boldsymbol{u}_{x_{i}}$. These again are connected to the spacecraft's reference system components $X_{j}$ through a matrix describing the rigid rotation of the comet

$x_{i}=\sum_{j=1}^{3} \alpha_{j i}\left(l_{\mathrm{c}}, g_{\mathrm{c}}, J_{\mathrm{c}}\right) X_{j}, \quad \alpha_{i j}=\boldsymbol{u}_{X_{i}} \cdot \boldsymbol{u}_{x_{j}}$,

enabling the operation of Eq. (2). Since simulations of 67P's rotation state show that it is possible, although not common, for the rotation to get excited through an orbital run (Gutiérrez et al. 2003), the matrix $\alpha$ must accommodate complex rotation, as indicated by its arguments. As illustrated in Fig. $1, g_{\mathrm{c}}$ is the so-called precession angle, i.e. the node along the plane normal to the spin $\boldsymbol{G}_{\mathrm{c}}$, of the nucleus' equator with respect to the reference $X$-axis. The polhode angle $l_{\mathrm{c}}$ is the angular distance of the principal $x$-axis from the spin plane, and is measured along the nucleus equator, defined by the principal $x$ - and $y$-axes. At last, $J_{\mathrm{c}}$ is the inclination of the body equator with respect to the plane normal to the comet's rotational spin. Also according to Gutiérrez et al. (2003), torques acting on 67P are negligible for long periods of time during the Rosetta mission, making the simulation of the angles above, the two first partially defining the so-called Andoyer coordinates, very easy. See Kinoshita (1972) for their differential equations. These then appear in Eq. (2) as explicit time dependencies. $\alpha$ is straightforward to derive, but can also be found in Beletskii (1966) with the help of the variable identifications given in Mysen (2004).

For the shape of the comet nucleus the hypothesis of Muinonen (1998) is adopted where the surface is described by multivariate normal statistics. The surface correlation angle is set to a low (Muinonen \& Lagerros 1998) value $\Gamma_{\mathrm{c}}=0.4$, i.e. high irregularity, while the standard deviation of the radii is defined to be $\sigma_{\mathrm{c}}=0.2 r_{\mathrm{c}}$ with $r_{\mathrm{c}}$ as the mean radius of the comet. A realization of these parameters is shown in Fig. 2, henceforth adopted as the nucleus.

If we, for simplicity only, define the mass density to be constant, an assumption which worked well for Eros

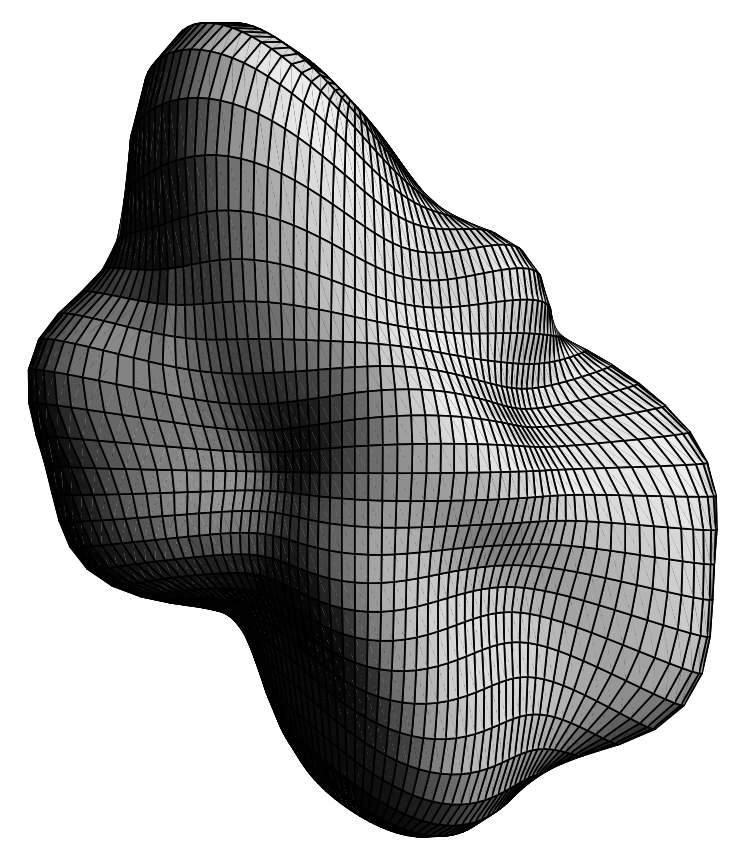

Fig. 2. The adopted Gaussian comet nucleus.

(Miller et al. 2002), the coefficients $c_{n m}$ and $s_{n m}$ of the expansion (1) can be calculated according to (Heiskanen \& Moritz 1967)

$$
\begin{aligned}
\left\{\begin{array}{c}
c_{n m} \\
s_{n m}
\end{array}\right\}= & \gamma_{n m}\left\{\begin{array}{l}
\bar{c}_{n m} \\
\bar{s}_{n m}
\end{array}\right\}=-\left(2-\delta_{0 m}\right) \frac{(n-m) !}{(n+m) !} \\
& \times \sum_{i} \frac{m_{i}}{m_{\mathrm{c}}}\left(\frac{r_{i}}{r_{\mathrm{c}}}\right)^{n}\left\{\begin{array}{l}
C_{n m}\left(\lambda_{i}, \beta_{i}\right) \\
S_{n m}\left(\lambda_{i}, \beta_{i}\right)
\end{array}\right\}
\end{aligned}
$$

where

$\gamma_{n m}=\sqrt{\frac{2(2 n+1)(n-m) !}{\left(1+\delta_{0 m}\right)(n+m) !}}$

is a normalization factor so that the square of the normalized spherical harmonic functions $\bar{C}_{n m}$ and $\bar{S}_{n m}$

$\left\{\begin{array}{c}C_{n m} \\ S_{n m}\end{array}\right\}=\frac{1}{\gamma_{n m}}\left\{\begin{array}{l}\bar{C}_{n m} \\ \bar{S}_{n m}\end{array}\right\}=P_{n m}(\sin \beta)\left\{\begin{array}{c}\cos m \lambda \\ \sin m \lambda\end{array}\right\}$

equal one when averaged over all directions. $\delta_{0 m}$ is the Kronecker delta. The sum is over all 16601 equidistant, equal point masses $m_{i}$ which we have defined the nucleus to consist of. For triaxial ellipsoids with uniform mass density, $c_{n m}=0$ if $n$ or $m$ odd and $s_{n m}=0$ (Rossi et al. 1999), making the corresponding terms measures of irregularity in our case. However, as long as the body-fixed system is mass centered and aligned with the principal axes, $c_{21}=s_{21}=s_{22}=0$, regardless of density distribution.

Some normalized values are given in Table 1. Since they in general do not vanish with increasing degree, the gravitational expansion (1) converges, roughly speaking, outside $r \sim r_{\mathrm{c}}$ (Garmier \& Barriot 2001). The upper row in Table 1 corresponds, in this case, to so-called short-axis rotational mode (SAM) of the nucleus, while the lower represents the case of rotation around the nucleus' long axis (LAM). $e_{\mathrm{c}}$ is the important triaxiality parameter determining the convergence of 
Table 1. Coefficients of the gravitational field Eq. (1).

\begin{tabular}{cccccc}
\hline \hline$e_{\mathrm{c}}$ & $\bar{c}_{20}$ & $\bar{c}_{22}$ & $\bar{c}_{30}$ & $\bar{c}_{32}$ & $\bar{s}_{33}$ \\
\hline 0.76 & 0.054 & -0.063 & -0.0098 & -0.0063 & -0.048 \\
0.075 & -0.082 & 0.015 & 0.040 & 0.036 & -0.010 \\
\hline
\end{tabular}

Table 2. Nominal 67P characteristics.

\begin{tabular}{ccc}
\hline \hline$m_{\mathrm{c}}$ & $r_{\mathrm{c}}$ & $2 \pi C G_{\mathrm{c}}^{-1}$ \\
\hline $10^{13} \mathrm{~kg}$ & $1.98 \mathrm{~km}$ & $12.3 \mathrm{~h}$ \\
\hline
\end{tabular}

the Andoyer variables' Fourier expansion in their action-angles (Kinoshita 1972).

Table 2 shows the nucleus mass $m_{\mathrm{c}}$ and the other parameters which are used in later simulations and can, on the basis of Lamy et al. (2003), Rickman et al. (1987) and Davidsson \& Gutiérrez (2005), be considered nominal. $C$ is the moment of inertia along the principal axis of rotation. If the rotation is uniaxial, the nucleus' rotational velocity $\omega$ coincides with this axis. For excited rotation, $\omega$ circulates around the principal axis of rotation in the nucleus corotating frame. As a consequence, both $g_{\mathrm{c}}$ and $l_{\mathrm{c}}$ are defined to never librate, but always circulate. The moments of inertia along the two other principal axes of the nucleus are $A$ and $B$. If $C$ is the largest of the three (SAM), we define the order $C>B>A$. If $C$ is smallest (LAM), then $C<B<A$ by definition.

\section{The Jacobi function}

Considering gravitation only, the motion of a probe can be constrained by a function $\chi$ which is an integral for uniaxial rotation. Below we shall see to what extent its constancy is violated in the general rigid body case, also treated in Scheeres et al. (1996).

\subsection{Definition}

Following Goldstein (1980), the velocity of the probe relative to the cometocentric, nucleus corotating system is

$\dot{\boldsymbol{r}}_{\mathrm{b}} \equiv \sum_{i=1}^{3} \boldsymbol{u}_{x_{i}} \dot{x}_{i}=\dot{\boldsymbol{r}}-\boldsymbol{\omega} \times \boldsymbol{r}$

where $\omega$ is the comet's angular velocity as before. If we take the time derivative of both sides, and then reapply the original equation, the relation

$\ddot{\boldsymbol{r}}_{\mathrm{b}}+\omega \times \dot{\boldsymbol{r}}_{\mathrm{b}}=\ddot{\boldsymbol{r}}-\dot{\omega} \times \boldsymbol{r}-\omega \times\left(\dot{\boldsymbol{r}}_{\mathrm{b}}+\omega \times \boldsymbol{r}\right)$

or

$$
\begin{aligned}
\ddot{\boldsymbol{r}}_{\mathrm{b}} & \equiv \sum_{i=1}^{3} \boldsymbol{u}_{x_{i}} \ddot{x}_{i} \\
& =\ddot{\boldsymbol{r}}-\omega \times(\boldsymbol{\omega} \times \boldsymbol{r})-2 \omega \times \dot{\boldsymbol{r}}_{\mathrm{b}}-\dot{\boldsymbol{\omega}} \times \boldsymbol{r},
\end{aligned}
$$

is obtained. For several important effects, like gravitation, the force per mass unit $\boldsymbol{f}=\ddot{\boldsymbol{r}}$ is derivable from a potential $V$

$$
\begin{aligned}
\ddot{\boldsymbol{r}} & =\boldsymbol{f}=-\nabla V \equiv-\sum_{i=1}^{3} \boldsymbol{u}_{X_{i}} \frac{\partial V}{\partial X_{i}} \\
& =-\sum_{i, j, k=1}^{3} \alpha_{i j} \boldsymbol{u}_{x_{j}} \alpha_{i k} \frac{\partial V}{\partial x_{k}}=-\sum_{k=1}^{3} \boldsymbol{u}_{x_{k}} \frac{\partial V}{\partial x_{k}} .
\end{aligned}
$$

The latter equality follows from the fact that the reference system and the nucleus corotating system is linked by an orthogonal transformation, i.e. $\alpha^{T} \alpha=1$, see Eq. (3), where $T$ here denotes the transpose matrix.

The first term of Eq. (9) after $\ddot{\boldsymbol{r}}$ is the centrifugal acceleration, derivable from the potential

$W=-\frac{1}{2}(\omega \times \boldsymbol{r})^{2}$.

Multiplying both sides of Eq. (9) with $\dot{\boldsymbol{r}}_{\mathrm{b}}$ removes the Coriolis component. Furthermore, since the gradient $\nabla$ is a rotational invariant, Eq. (10), and the gravitational field is stationary in the corotating system

$\dot{\boldsymbol{r}}_{\mathrm{b}} \cdot \nabla V=\dot{V}, \quad V \equiv V_{0}+V_{1}$

This is, however, not the case for the centrifugal acceleration

$\dot{\boldsymbol{r}}_{\mathrm{b}} \cdot \nabla W=\dot{W}-\frac{\partial W}{\partial t}=\dot{W}+(\dot{\boldsymbol{\omega}} \times \boldsymbol{r}) \cdot(\boldsymbol{\omega} \times \boldsymbol{r})$.

Defining the Jacobi function as

$\chi \equiv \frac{1}{2} \dot{r}_{\mathrm{b}}^{2}+V+W$,

then yields for its time derivative

$\dot{\chi}=-(\dot{\omega} \times \boldsymbol{r}) \cdot\left(\dot{\boldsymbol{r}}_{\mathrm{b}}+\omega \times \boldsymbol{r}\right)=-(\dot{\omega} \times \boldsymbol{r}) \cdot \dot{\boldsymbol{r}}$.

Using a fundamental vector identity, and the definition of the probe's orbital angular momentum $\boldsymbol{G} \equiv \boldsymbol{r} \times \dot{\boldsymbol{r}}$,

$(\dot{\omega} \times \boldsymbol{r}) \cdot \dot{\boldsymbol{r}}=\dot{\omega} \cdot(\boldsymbol{r} \times \dot{\boldsymbol{r}}) \equiv \dot{\omega} \cdot \boldsymbol{G}$,

and therefore

$\chi(t)=\chi\left(t_{0}\right)-\left.\boldsymbol{\omega} \cdot \boldsymbol{G}\right|_{t_{0}} ^{t}+\int_{t_{0}}^{t} \mathrm{~d} t \boldsymbol{\omega} \cdot \dot{\boldsymbol{G}}$

follows for the Jacobi function of a probe in an orbit around a complex rotating body.

\subsection{The modified Jacobi function}

Suppose that the probe interacts chaotically with the comet, for instance near its pericenter, altering the spacecraft's angular momentum with a vector $\Delta \boldsymbol{G}=\boldsymbol{G}(t)-\boldsymbol{G}\left(t_{0}\right)$. Furthermore, assume that the interaction times $t_{X_{k}}$

$\int_{t_{0}}^{t} \mathrm{~d} t \omega_{X_{k}} \dot{G}_{X_{k}}=\omega_{X_{k}}\left(t_{X_{k}}\right) \Delta G_{X_{k}}, \quad t_{0}<t_{X_{k}}<t$

introduced more for notational convenience, can be defined. Eq. (18) is valid for a wide range of function combinations, but 
not as a mathematical statement. For the scenarios which motivate this paper, the orbital angular momentum changes aperiodically, and Eq. (18) is more true in general. Inserting Eq. (18) into Eq. (17) for several interactions $N$

$\boldsymbol{G}(t)=\boldsymbol{G}\left(t_{0}\right)+\sum_{i=1}^{N} \Delta \boldsymbol{G}_{i}$,

we obtain

$$
\begin{aligned}
\chi(t)-\chi\left(t_{0}\right)= & -\sum_{i=1}^{N} \sum_{j=1}^{2} \Delta G_{X_{j}, i}\left[\omega_{X_{j}}(t)-\omega_{X_{j}}\left(t_{X_{j}, i}\right)\right] \\
& -\sum_{j=1}^{2} G_{X_{j}}\left(t_{0}\right)\left[\omega_{X_{j}}(t)-\omega_{X_{j}}\left(t_{0}\right)\right]
\end{aligned}
$$

by noting that the angular velocity's component along the $Z$-axis, $\omega_{Z}$, is constant for a freely rotating rigid body. According to Eq. (20), the Jacobi function deviates from its epoch value by a series of periodic terms, the last two being independent of the orbital evolution.

Without drawing any conclusions yet regarding the terms containing $\Delta G_{X_{j}}$, it is evident that the parts of Eq. (20) which are independent of the orbital evolution could be significant for inclined orbits. However, the components in question can be removed by converting the comet-fixed probe velocity to its more absolute equivalent

$$
\begin{aligned}
\chi & =\frac{1}{2}(\dot{\boldsymbol{r}}-\boldsymbol{\omega} \times \boldsymbol{r})^{2}-\frac{1}{2}(\boldsymbol{\omega} \times \boldsymbol{r})^{2}+V \\
& =\frac{1}{2} \dot{\boldsymbol{r}}^{2}+V-\boldsymbol{\omega} \cdot \boldsymbol{G},
\end{aligned}
$$

which inserted into Eq. (17) with the use of expansions (18), results in

$\Omega(t)-\Omega\left(t_{0}\right)=\sum_{i=1}^{N} \sum_{j=1}^{2} \Delta G_{X_{j}, i} \omega_{X_{j}}\left(t_{X_{j}, i}\right)$,

where the modified Jacobi function is

$\Omega \equiv \frac{1}{2} \dot{\boldsymbol{r}}^{2}+V-\omega_{Z} G \cos I$

with $I$ as the probe orbit's inclination with respect to the spin plane. If the nucleus rotation is one-axial, then $\Omega=\chi$.

\subsection{A pseudo-integral}

We will now estimate the variations Eq. (22) relative to $\Omega$ 's possible range

$\frac{\Delta \Omega}{\Omega} \sim \frac{\omega_{X_{k}}\left(t_{X_{k}}\right)}{\omega_{Z}} \frac{\Delta G_{X_{k}}}{G}$

where it has been assumed that the maximum of the third of Eq. (23)'s terms, and not the two first representing the orbital energy, is dominant. That is, we assume that the nucleus is not much more massive or much more slowly rotating than our nominal one. However, with the anlysis presented below, estimates of $\Omega$ 's absolute change for such cases should be possible to produce. Following Eq. (24), the conditions for which $\Omega$ is

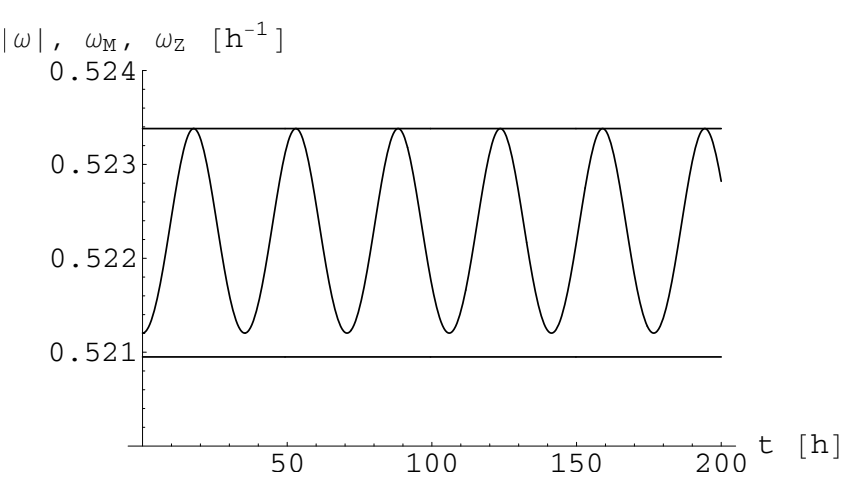

Fig. 3. The simulated $|\omega|=\sqrt{\omega^{2}}$ (variable curve) and $\omega_{Z}$ (lower line) are plotted for the nucleus in SAM. $\omega_{Z}$ and $\omega_{\mathrm{M}}$ from Eqs. (26) and (27) are also included, but the former is not visible since it concides with its simulated value.

nearly conserved can be found. First consider $\omega_{X}\left(t_{X}\right) / \omega_{Z}$ since the analysis for $\omega_{Y}\left(t_{Y}\right) / \omega_{Z}$ is identical with exactly the same quantitative conclusions.

If one makes the conservative assumption that $\omega_{X}$ 's value at the interaction time is not correlated with $\Delta G_{X}$, the former can take on all accessible values. These include zero, a mean $\bar{\omega}$, and a maximum $\omega_{m}$, where the range $\omega_{X}\left(t_{X}\right) \in(0, \bar{\omega})$ is more relevant since the chaotic interactions generally take place over typically a comet rotation period for the nominal parameters. Identifying $\omega_{m}^{2}=\omega_{M}^{2}-\omega_{Z}^{2}$ where

$\omega_{\mathrm{M}}^{2}=\max \left[\omega_{x}^{2}+\omega_{y}^{2}+\omega_{z}^{2}\right], \quad \omega_{Z}=\frac{2 T_{\mathrm{c}}}{G_{\mathrm{c}}}$,

with $T_{\mathrm{c}}$ as the nucleus' rotational kinetic energy, it is straightforward to derive

$\omega_{Z}=\frac{1}{\varrho} \tilde{\omega} \frac{2 \varrho e_{\mathrm{c}}+k^{2}\left(1-e_{\mathrm{c}}\right)}{2 e_{\mathrm{c}}+k^{2}\left(1-e_{\mathrm{c}}\right)}$

$\omega_{M}=\frac{1}{\varrho} \tilde{\omega} \sqrt{\frac{2 \varrho^{2} e_{\mathrm{c}}+k^{2}\left(1-e_{\mathrm{c}}\right)}{2 e_{\mathrm{c}}+k^{2}\left(1-e_{\mathrm{c}}\right)}}$

with the use of the principal axis components $\omega_{x_{k}}$ of Kinoshita (1972). Above $\varrho=A / C, \tilde{\omega}=G_{\mathrm{c}} / C$, and $k$ is the elliptic modulus of free rotation. For motion on the separatrix separating the rotational modes $k=1$, while $k=0$ means uniaxial rotation if $B$, the intermediary moment of inertia, is not identical to $A$, see for instance Hitzl \& Breakwell (1971). Based on a number of simulations of the equations of motion for a freely rotating rigid body, both Eqs. (26) and (27) have been found to be correct. Figure 3 shows the results of such a simulation for the nominal nucleus in SAM at an excited $k=0.500$.

The process of obtaining $\bar{\omega}$ is more elaborate. The mean used for its calculation is defined to be

$\lim _{T \rightarrow \infty} \frac{1}{T} \int_{0}^{T} \mathrm{~d} t=\frac{1}{(2 \pi)^{2}} \int_{0}^{2 \pi} \mathrm{d} \tilde{l}_{\mathrm{c}} \int_{0}^{2 \pi} \mathrm{d} g_{\mathrm{c}}$,

where the equality follows if the rotation is approximately torque free. $\tilde{l}_{\mathrm{c}}$ is one of the Andoyer action-angles, having a constant time derivative for free rigid rotation. Actually, the action-angle $\tilde{g}_{\mathrm{c}}$ should also have been used above, but the 
integration over the precession angle $g_{\mathrm{c}}$ is equivalent since (Kinoshita 1972)

$g_{\mathrm{c}}=\tilde{g}_{\mathrm{c}}+\sum_{m=1}^{\infty} c_{m}\left(e_{\mathrm{c}}, k\right) \cos 2 m \tilde{l}_{\mathrm{c}}$.

Using the rotation $\alpha$ of Eq. (3), which can be separated into

$\alpha_{i j}=\tilde{\alpha}_{i j}^{(c)} \cos g_{\mathrm{c}}+\tilde{\alpha}_{i j}^{(s)} \sin g_{\mathrm{c}}, \quad i \neq 3$

results in

$\omega_{X_{1}}=\gamma\left(l_{\mathrm{c}}\right) \cos g_{\mathrm{c}}+\delta\left(l_{\mathrm{c}}\right) \sin g_{\mathrm{c}}$

$\omega_{X_{2}}=-\delta\left(l_{\mathrm{c}}\right) \cos g_{\mathrm{c}}+\gamma\left(l_{\mathrm{c}}\right) \sin g_{\mathrm{c}}$,

where, for instance

$\gamma\left(l_{\mathrm{c}}\right)=\sum_{i=1}^{3} \tilde{\alpha}_{1 i}^{(c)} \omega_{x_{i}}, \quad \delta\left(l_{\mathrm{c}}\right)=\sum_{i=1}^{3} \tilde{\alpha}_{1 i}^{(s)} \omega_{x_{i}}$,

and therefore

$\frac{1}{2 \pi} \int_{0}^{2 \pi} \mathrm{d} g_{\mathrm{c}} \omega_{X}^{2}=\frac{1}{2}\left(\gamma^{2}+\delta^{2}\right)$.

The remaining integration can be performed after a lengthy, but straightforward formulation of $\gamma^{2}+\delta^{2}$ in principal axis spin components (Kinoshita 1972), which in free rigid body motion are pure functions of $\tilde{l}_{\mathrm{c}}$. Consult Byrd \& Friedman (1971) for the relevant integrals. The end result is

$\bar{\omega} \equiv \sqrt{\lim _{T \rightarrow \infty} \frac{1}{T} \int_{0}^{T} \mathrm{~d} t \omega_{X}^{2}}=\frac{1}{2 \sqrt{2}} \tilde{\omega}|1-1 / \varrho| \psi\left(e_{\mathrm{c}}, k\right)$

$\psi=\frac{2}{\bar{b}\left(1+e_{\mathrm{c}}\right)} \sqrt{\left(1+\bar{b} e_{\mathrm{c}}\right)\left[\frac{E}{K} \bar{b}\left(1-e_{\mathrm{c}}\right)+\bar{b} e_{\mathrm{c}}-1\right]}$.

where

$\bar{b}\left(e_{\mathrm{c}}, k\right)=\frac{2 e_{\mathrm{c}}+k^{2}\left(1-e_{\mathrm{c}}\right)}{e_{\mathrm{c}}\left[2-k^{2}\left(1-e_{\mathrm{c}}\right)\right]}$,

and $K$ and $E$ are the complete Jacobian elliptic integrals of the first and the second kind, respectively

$K(k)=\int_{0}^{\pi / 2} \frac{\mathrm{d} \theta}{\sqrt{1-k^{2} \sin ^{2} \theta}}$

$E(k)=\int_{0}^{\pi / 2} \mathrm{~d} \theta \sqrt{1-k^{2} \sin ^{2} \theta}$.

As for Eqs. (26) and (27), Eq. (34) has been found to be perfectly consistent with integrals of simulated solutions.

Plotted as Fig. 4 are the $\bar{\omega} / \omega_{Z}$ and $\omega_{m} / \omega_{Z}$ ratios for the nucleus Fig. 2 in SAM (dashed) and LAM (solid). The solid curve is typical for low triaxial modes, rapidly rising to a large value for increasing excitation $k$. However, it must be stressed that the lower $e_{\mathrm{c}}$, the higher (the maximum of) $J_{\mathrm{c}}$ must be in order to produce a specific $k$. If $k$ is fixed at 0.5 , the vertical line of Fig. 4 , the iso-contours of the ratio $\bar{\omega} / \omega_{Z}$ can be plotted in $\varrho-e_{\mathrm{c}}$ space, Fig. 5. Clearly, nuclei with high triaxiality, the intermediary moment of inertia $B$ being closer to $C$ than $A$,

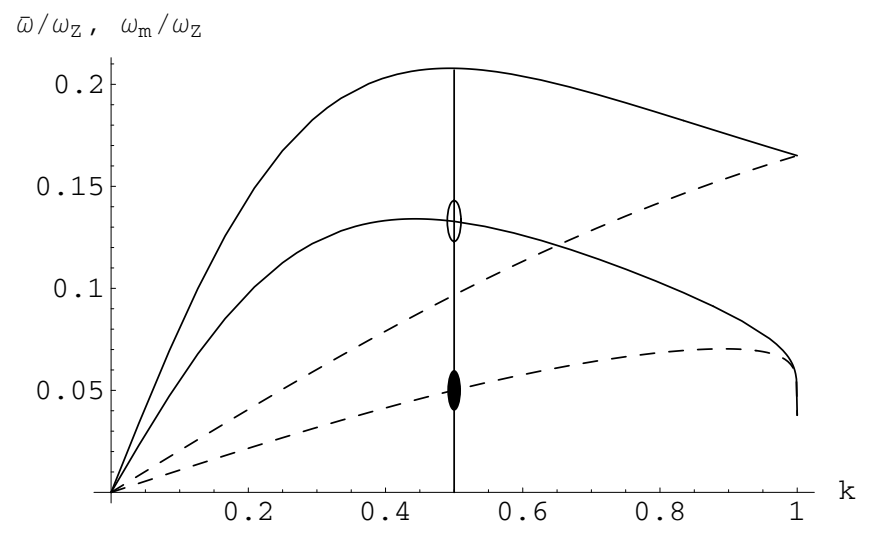

Fig. 4. $\bar{\omega} / \omega_{Z}$ (lower) and $\omega_{m} / \omega_{Z}$ (upper) as a function of the rotation excitation parameter $k$ for the low (solid) and high (dashed) triaxiality mode, Table 1.

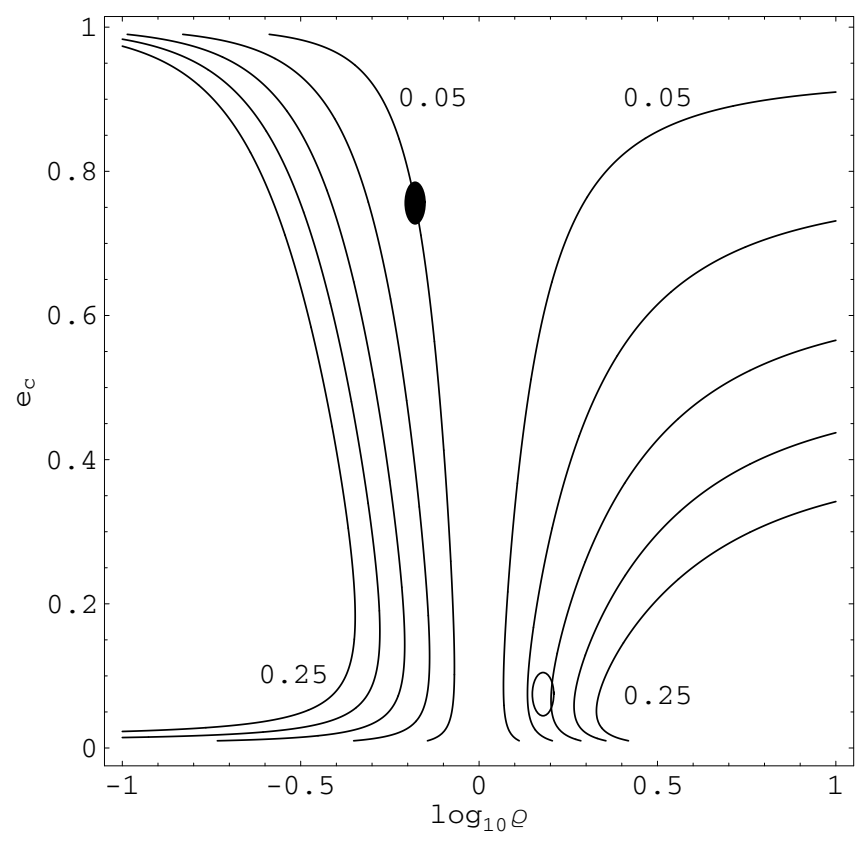

Fig. 5. Level sets of $\bar{\omega} / \omega_{Z}$ in inertia ratio $\varrho=A / C$ and triaxiality space. The values at the curves increase from 0.05 to 0.25 with step 0.05 from the center to the left and right. Also included are the ellipses of Fig. 4, representing the different modes of the nucleus on Fig. 2 at $k=0.5$.

which also are elongated, $\varrho>1$, have small upper boundaries on the relative change of $\Omega$.

As for the second factor of Eq. (24), we begin by observing that the expansion (1) can be separated into an $r=r(f)$ and a $u=f+g$ dependency for all orders $n$, with $f$ and $g$ as the true anomaly of the orbiter and the orbit's argument of pericenter (Murray \& Dermott 1999), respectively. More specifically, for the $n$ 'th harmonics

$V_{1}^{(n)}=\frac{\mu_{\mathrm{c}}}{r}\left(\frac{r_{\mathrm{c}}}{r}\right)^{n} Y_{n}(u)$

which yields

$\dot{G}^{(n)}=-\frac{\partial V_{1}^{(n)}}{\partial g}=-\frac{\mu_{\mathrm{c}}}{r}\left(\frac{r_{\mathrm{c}}}{r}\right)^{n} \frac{\partial Y_{n}}{\partial u}$ 
According to Scheeres et al. (1996), changes in, for instance, the orbit's angular momentum can be estimated holding the eccentricity and semi-major axis constant and equal to their initial values. Assuming that the changes are aperiodic, we then have

$\Delta G^{(n)}=-\mu_{\mathrm{c}} r_{\mathrm{c}}^{n} \int_{t_{0}}^{t} \mathrm{~d} t \frac{1}{r^{n+1}} \frac{\partial Y_{n}}{\partial u}$,

or

$$
\frac{\Delta G^{(n)}}{G}=-\frac{r_{\mathrm{c}}^{n}}{q(1+e)} \overline{\left(\frac{\partial Y_{n}}{\partial u}\right)} \int_{-\pi}^{\pi} \mathrm{d} f \frac{1}{r^{n-1}}
$$

for the change from apocenter at time $t_{0}$ to the next apocenter at time $t$, where the relation $\mathrm{d} t=\mathrm{d} f r^{2} / G$ and $G=\sqrt{\mu_{\mathrm{c}} q(1+e)}$ have been used. Here $e$ is the orbit eccentricity and $q=a(1-e)$ is the pericenter distance, with $a$ as the orbit's semi-major axis. The bar indicates that the function is to be evaluated somewhere along the orbit, but the simplification

$\left|\frac{\partial Y_{n}}{\partial u}\right| \sim\left|Y_{n}\right| \lesssim \max \left[\left|\bar{c}_{n m}\right|,\left|\bar{s}_{n m}\right|\right] \equiv \bar{c}_{n}$

is adopted.

The integrals of Eq. (42) are easily evaluated

$\int_{-\pi}^{\pi} \mathrm{d} f r^{-1}=\frac{2 \pi}{q(1+e)}$

$\int_{-\pi}^{\pi} \mathrm{d} f r^{-2}=\frac{2 \pi\left(1+e^{2} / 2\right)}{q^{2}(1+e)^{2}}, \quad$ etc.

and hence typically

$\left|\frac{\Delta G^{(n)}}{G}\right| \lesssim 2 \pi \bar{c}_{n}\left[\frac{r_{\mathrm{c}}}{q(1+e)}\right]^{n}$,

amounting to a low total of approximately 0.05 at a close $q=$ $2.5 r_{\mathrm{c}}$ with $e=0.5$. Now, the component of the orbital angular momentum along the spin axis of the nucleus, $H=G \cos I$, can be treated in exactly the same way

$\dot{H}^{(n)}=-\frac{\partial V_{1}^{(n)}}{\partial h}=-\frac{\mu_{\mathrm{c}}}{r}\left(\frac{r_{\mathrm{c}}}{r}\right)^{n} \frac{\partial Y_{n}}{\partial h}$,

where $h$ is the orbit's longitude of ascending node. This implies that $\boldsymbol{G}$ not only experiences a limited change in magnitude, Eq. (46), but also in direction. It follows that Eq. (46) with $\Delta G^{(n)} \rightarrow \Delta G_{X, Y}^{(n)}$, as in Eq. (24), also is valid.

We have now argued that both factors of the product representing the change in what we have called the modified Jacobi function, are small in general. Therefore, if the nucleus geometry is not too extreme and the orbit is not very close, we can conclude that $\Delta \Omega / \Omega \ll 1$ even when the change in actions are aperiodic and large.

To illustrate this, the motion of a probe under the influence of the adopted nucleus' gravitational field is simulated as described in Sect. 2. The nucleus is assumed to rotate in the long-axis or short-axis mode with $k=0.500$ where the former, consistent with these studies, represents a worst-case scenario. Included as Fig. 6 is the variation of the modified Jacobi function of the probe, relative to its starting value, as a function of

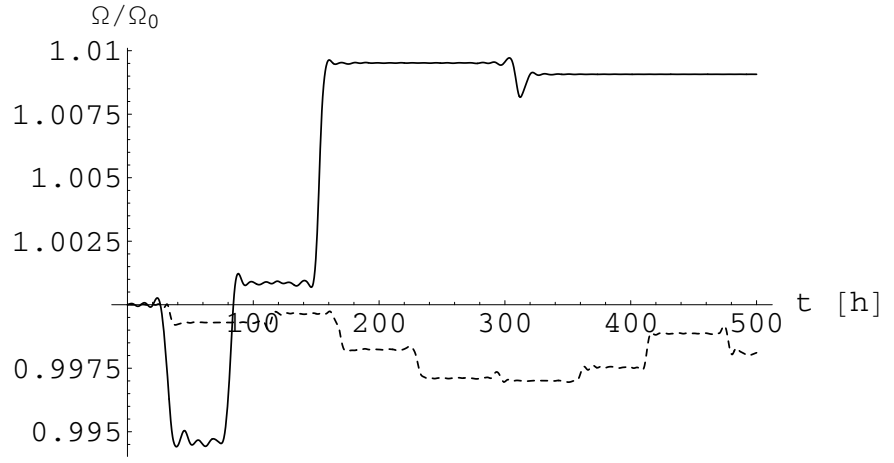

Fig. 6. The relative variation of a cometary orbiter's modified Jacobi function in an inclined and prograde orbit, as determined by the nucleus rotation. The dashed curve is for motion around the high triaxiality mode (SAM), while the solid one is for LAM.

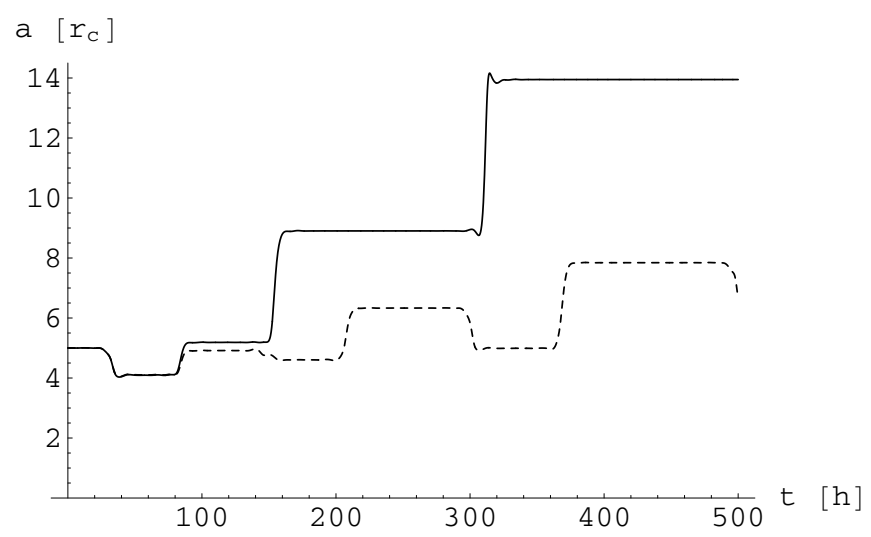

Fig. 7. Variation of an orbiting probe's semi-major axis $a$ in mean comet radii $r_{\mathrm{c}}$ for LAM as a function of time in hours. The dashed curve is calculated with components of the gravitational field up to and including order $n=5$, while the solid one is for up to and including order $n=6$.

time in hours for a gravitational field Eq. (1) up to and including order $n=6$. At $t_{0}=0$, the orbiting spacecraft is at its trajectory's apocenter $r=7.5 r_{\mathrm{c}}$, heading towards its initial pericenter at $q_{0}=2.5 r_{\mathrm{c}}$ in a prograde and slightly inclined orbit, with respect to the nucleus spin plane. This choice is motivated by the exceptionally large variations of $\Omega$ for LAM, relative to other orbits. The changes are nevertheless quite limited, and most certainly so in comparison to the variations in semi-major axis, Fig. 7. Also included is a plot of $\Delta G_{X}$, Fig. 8. Although there is little difference in semi-major axis of the two orbits in Fig. 7 for the first $80 \mathrm{~h}$, long-term predictions are clearly difficult for close orbits around our adopted irregular nucleus. The used simulation algorithms are seen to preserve the Jacobi function to acceptable precision for uniaxial rotation.

\section{Impact avoidance}

As already mentioned, the conservation of $\chi$ has been used to constrain the motion of a probe in a prograde orbit. That is, for a negative $\chi_{0}, \dot{r}_{\mathrm{b}}^{2}$, as given by Eq. (14), could be less than zero at some distance close to the comet nucleus, clearly defining 
$\left(G_{x}(t)-G_{x}(0)\right) / G(t)$

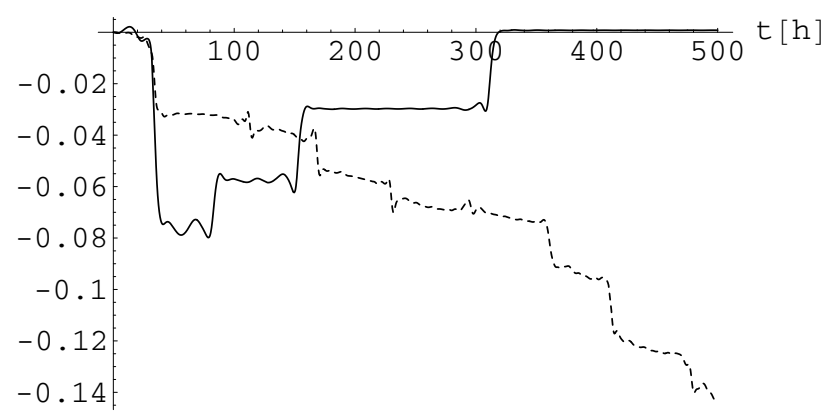

Fig. 8. The relative spin component alterations $\left[G_{X}(t)-G_{X}(0)\right] G(t)^{-1}$ for LAM (solid) or SAM (dashed). The order of the gravitational field is $n=6$.

a region which is prohibited for the orbiter. At the boundaries of these regions $\left|\dot{\boldsymbol{r}}_{\mathrm{b}}\right|=0$, and they are therefore referred to as zero-velocity curves. Often, as is most convenient, the analysis is made in the Cartesian components of the nucleus corotating reference system. Here however, we will refrain from doing so for two reasons. First, $\chi$ in Eq. (14) could have large periodic variations for inclined orbits, Eq. (20), and secondly, the centrifugal $W$ of Eq. (11) adds in general another dimension to the spatial analysis, time. Instead, we will characterize the unaccessible regions in terms of the probe's orbital elements as a function of the initial orbit, as is most natural for a conserved $\Omega$.

Prograde orbits are renowned for their unstable nature (see for instance Chauvineau et al. 1993), a property which indicates that the resonance widths are large for these trajectories. It is therefore of great interest that the implications of a conserved $\Omega$ can be quite strict for such orbits.

$\Omega=\Omega_{0} \equiv \Omega\left(t_{0}\right)$ in orbital elements corresponds to a Tisserand-like relation

$-\frac{1}{2 q}(1-e)-\omega_{Z}^{\prime} \sqrt{q(1+e)}=\Omega_{0}^{\prime}$,

which is valid for all eccentricities (Roy 1998). Here

$\Omega^{\prime} \equiv \frac{\Omega}{\mu_{\mathrm{c}}}, \quad \omega_{Z}^{\prime} \equiv \frac{\omega_{Z} \cos I}{\sqrt{\mu_{\mathrm{c}}}}$

with $I$ as the inclination of the current orbit with respect to the plane normal to $\boldsymbol{G}_{\mathrm{c}}$. Although the gravitational field modulated by the nucleus rotation is the very cause for the aperiodic variations in the orbiter's actions, its effect on the conservation law above has been neglected. The reason for this is that, unless the initial pericenter distance is $q<2 r_{\mathrm{c}}$, the induced corrections in closest approach distance, $\Delta q$, are in general smaller than those induced by the variation of $\Omega$, Fig. 6 , for our nominal nucleus. This can be confirmed with a later expression connecting $\Delta q / q$ to $\Delta \Omega^{(n)} / \Omega$ as induced by the higher harmonics of the gravitational field

$\left|\frac{\Delta \Omega^{(n)}}{\Omega}\right| \lesssim \frac{1}{\omega_{Z} \sqrt{\mu_{\mathrm{c}} q(1+e)}} \frac{\mu_{\mathrm{c}}}{q} \bar{c}_{n}\left(\frac{r_{\mathrm{c}}}{q}\right)^{n}$

where $\bar{c}_{n}$ is from Eq. (43), resulting in a total of $\lesssim 0.01$ for $q=2.5 r_{\mathrm{c}}$ and $e=0.5$.
Equation (48) defines curves in $e-q$ space on which the orbit must exist given its present inclination $I$. Moving the first term of Eq. (48)'s left hand side over to the right hand side and squaring, two eccentricity roots are obtained

$e^{ \pm}=w-1+\omega_{Z}^{\prime 2} q^{3} \pm 2 \sqrt{\omega_{Z}^{\prime 2} q^{3} w}$

where

$w \equiv 2+2 \Omega_{0}^{\prime} q+\omega_{Z}^{\prime 2} q^{3}$.

Substituting these back into Eq. (48) which we multiply by $-q$, and observing that

$\sqrt{1+e^{ \pm}}=\left|\sqrt{w} \pm \sqrt{\omega_{Z}^{\prime 2} q^{3}}\right|$

we obtain

$\mp \sqrt{\omega_{Z}^{\prime 2} q^{3} w}-\omega_{Z}^{\prime 2} q^{3}$

$+\operatorname{sign}[\cos I]\left|\sqrt{\omega_{Z}^{\prime 2} q^{3} w} \pm \omega_{Z}^{\prime 2} q^{3}\right|=0$

with the upper sign for $e^{+}$, and the lower for $e^{-}$. If $\cos I<0$, Eq. (48) shows that $\Omega_{0}^{\prime}>0$ unless $\omega_{Z}^{\prime}$ is very small or the orbit is bound with $q \sim 0$, and we therefore have that

$\sqrt{\omega_{Z}^{\prime 2} q^{3} w}>\omega_{Z}^{\prime 2} q^{3}$

Under these circumstances, $e^{-}$is seen to be the only true solution of Eq. (48). Likewise, if $\cos I>0, \Omega_{0}^{\prime}<0$ unless the orbit is very hyperbolic, and it follows that

$\sqrt{\omega_{Z}^{\prime 2} q^{3} w}<\omega_{Z}^{\prime 2} q^{3}$

if $q>q_{ \pm} \equiv 1 /\left|\Omega_{0}^{\prime}\right|$. With these prerequisites, both roots $e^{ \pm}$make Eq. (54) true. If, on the other hand, this last criterion is not satisfied, i.e. $q<q_{ \pm}$, the prograde trajectory move on the $e^{+}$branch only as can be seen from Eq. (54).

Holding the inclination fixed, we see that for a specific pericenter distance $q>q_{ \pm}$, there exist two possible eccentricities for a prograde orbit as long as they are defined by Eq. (51). Now, if $\Omega_{0}^{\prime}<0, w(q)$ could be zero for some low $q=q^{*}$, which marks the transition from one eccentricity root to the other. Just below this pericenter distance $w(q)$ is negative and Eq. (48) therefore has no solutions for such $q$ 's. $q^{*}=q^{*}(I)$ is in other words the minimum value $q$ can take on at the given present inclination $I$. Setting the square root of Eq. (51), or $w$, to zero, we obtain this minimum approach distance. Substituting the trial solution

$q^{*}=\sqrt{\frac{8\left|\Omega_{0}^{\prime}\right|}{3}} \frac{\cos \vartheta}{\omega_{Z}^{\prime}}$

into the resulting third degree equation $w\left(q^{*}\right)=0$, yields

$\cos 3 \vartheta=-\sqrt{\frac{27}{8\left|\Omega_{0}^{\prime}\right|^{3}}} \omega_{Z}^{\prime}$

where the $\vartheta$ solution we are interested in, corresponding to the largest $q^{*}$ of Eq. (57), lies in the first quadrant. The two 


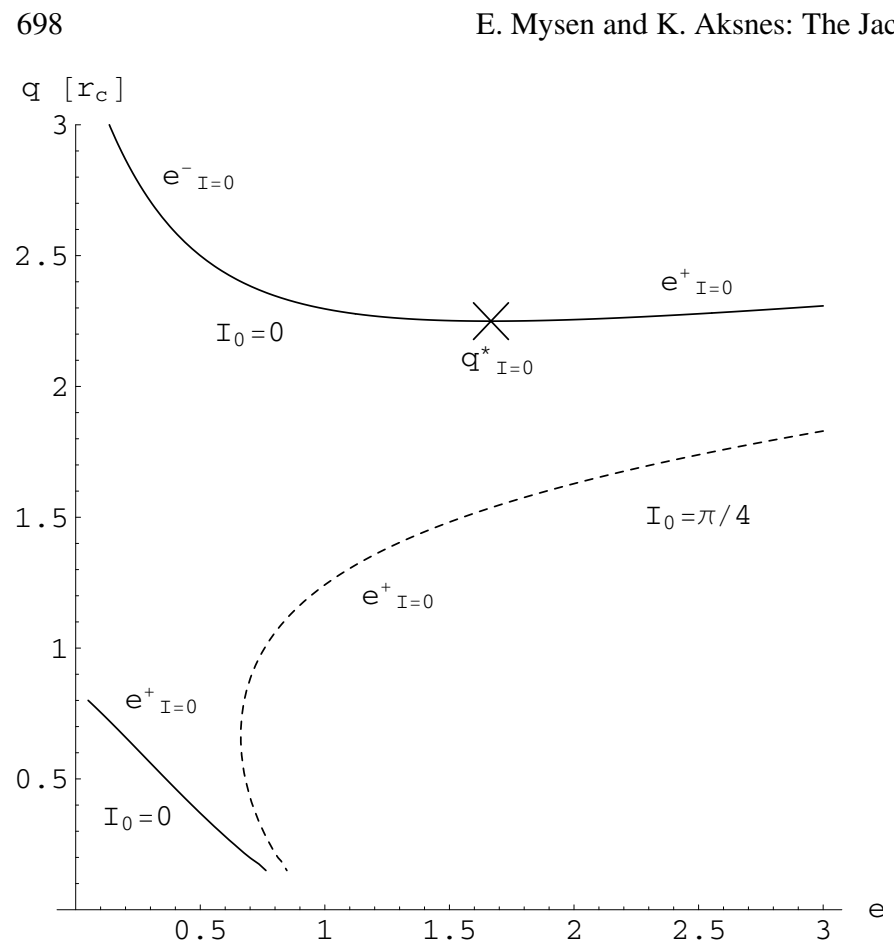

Fig. 9. Limiting contours for two prograde orbits.

other solutions are obtained by adding or subtracting $120^{\circ}$ from this $\vartheta$.

Clearly, a probe for which this scenario holds will never come closer to the comet nucleus than $q_{I=0}^{*}$ since $I=0$ represents the smallest minimum, corresponding to a scenario where the probe's orbit is perturbed in to the nucleus' spin plane from an initial inclination by a series of chaotic interactions. The exception here is when the solution type (57) no longer exists for all inclinations; i.e. when $|\cos 3 \vartheta|>1$ at $I=0$ or equivalently

$\left|\Omega_{0}^{\prime}\right|<\frac{3}{2} \omega_{Z, I=0}^{\prime 2 / 3}$

and, as can be reasoned, the only $q$ value which makes $w(q)$ zero is negative. That is, if Eq. (59) is true, the conservation of $\Omega$ alone cannot guarantee that the probe avoids collision with the nucleus for some end orbit lying close to the comet's spin plane. However, this scenario is circumvented if the probe orbit's initial inclination $I_{0}$ is chosen to be sufficiently low so that Eq. (59) is false. From the properties of $w(q)$, it can be shown that the $q^{*}$ solution we are interested in is always larger than $q_{ \pm}$ for at least as long as Eq. (59) is false.

All these aspects are illustrated in Fig. 9, showing the solutions of Eq. (48) at $I=0$ for two different orbit initial conditions and a nominal nucleus with $J_{\mathrm{c}}=0$. The solid curves represent the choice $I_{0} \equiv I\left(t_{0}\right)=0$, in this case violating Eq. (59), while the dashed segment is produced by an initial trajectory with $I_{0}=\pi / 4$ and Eq. (59) being true. Both have the initial $q_{0}=2.5 r_{\mathrm{c}}$ and $e_{0}=0.5$. Each contour in the plot has been identified as one of Eq. (51)'s branches. The orbit which started in the spin plane can never exist between the two solid lines, while the initially inclined trajectory can never cross into the lower right region bounded by the dashed curve. This last

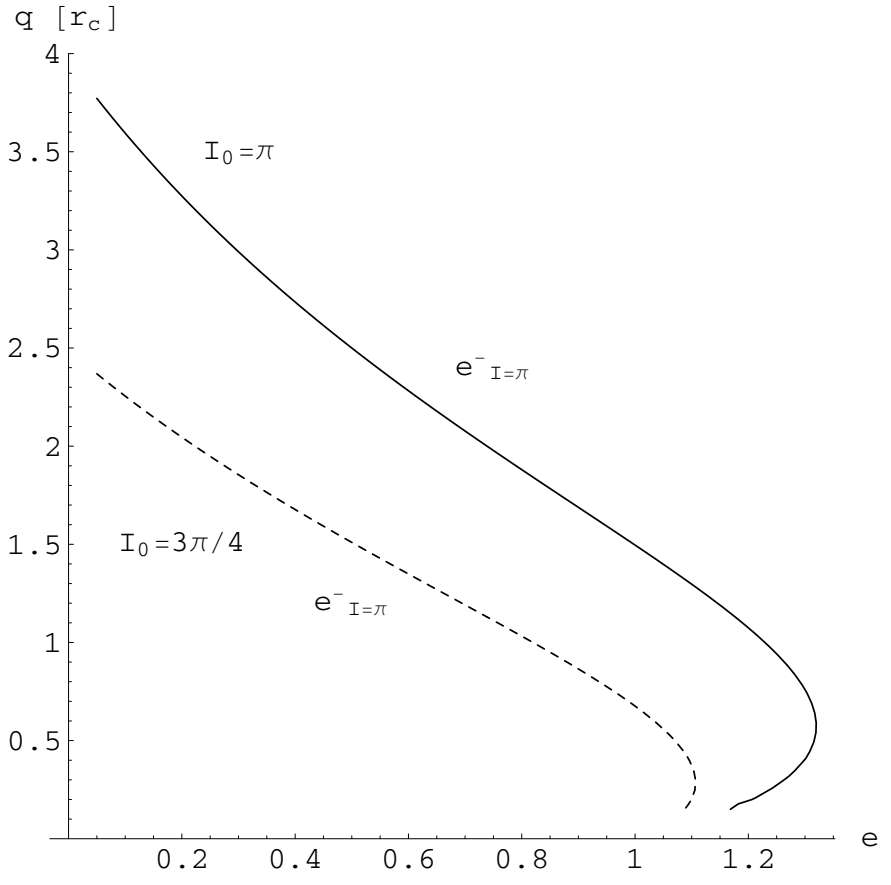

Fig. 10. Curves which mark the boundaries of the forbidden regions, lower left, for two retrograde orbits with different initial inclinations with respect to the spin plane.

conclusion rests on the fact that $e^{+}(q)$ close to $e=1(q=0)$ goes as

$e^{+}(q) \approx 1+2 \Omega_{0}^{\prime} q+2 \sqrt{2 \omega_{Z}^{\prime 2} q^{3}}, \quad \Omega_{0}^{\prime}<0$,

and therefore decreases less rapidly from $e=1$ with increasing $q$ for a low inclination than for a large one, only to return to a lower

$q_{h} \equiv q(e=1)=\frac{1}{2}\left(\frac{\Omega_{0}^{\prime}}{\omega_{Z}^{\prime}}\right)^{2}$.

$e_{I=0}^{-}$for the initially inclined case exists if $q>q_{ \pm}$, but is not included since it is negative. Also, at low $q$ 's, the irregularities of the gravitational field cannot be neglected, but the solutions for spherical symmetric gravitation are still used for solution identification purposes.

Likewise, the solutions of Eq. (48) at $I=\pi$ for two retrograde orbits with different initial inclinations are shown in Fig. 10. The dashed curve, marking the lower left region which is forbidden for the probe's orbit, represents the inclined $I_{0}=$ $3 \pi / 4$, as the solid line represents the boundary for the $I_{0}=\pi$ case. Other initial orbital parameters are as before. That the region in question is forbidden follows from the fact that $e^{-}(q)$ close to $e=1(q=0)$ goes as

$e^{-}(q) \approx 1+2 \Omega_{0}^{\prime} q-2 \sqrt{2 \omega_{Z}^{\prime 2} q^{3}}, \quad \Omega_{0}^{\prime}>0$,

and therefore increases less rapidly from $e=1$ with increasing $q$ for a large $|\cos I|$ than for a small one, only to return to a lower $q_{h}$, Eq. (61).

Since retrograde trajectories have $\Omega_{0}^{\prime}>0$, except for some special cases, $w(q)$ cannot be zero in the physical range of $q$ 's, 


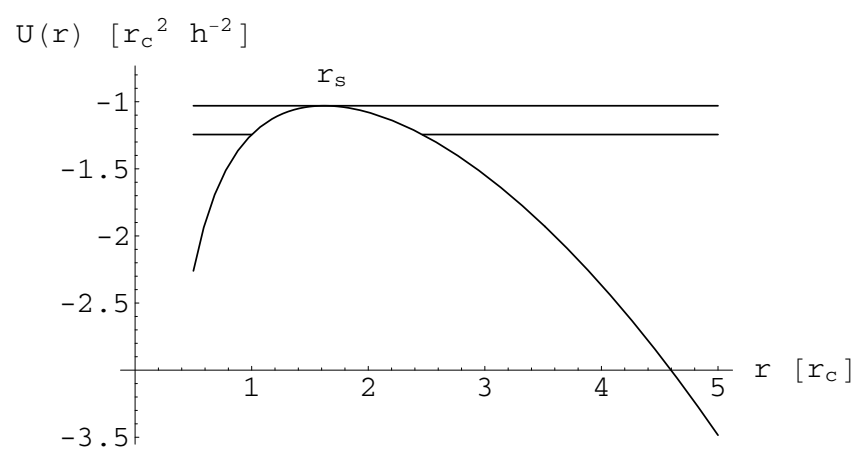

Fig. 11. The effective potential $U$ to zero order in the spin plane.

and there exists no minimum threshold on pericenter distance based on a constant $\Omega$. However, note that for the initially retrograde case $I_{0}=\pi$, the orbiting spacecraft's orbit must change into a hyperbolic trajectory above a distance $q_{h}$ on its inbound leg if it is to collide with the nucleus. Therefore, proper precautions can be taken by choosing $q_{h}$ to be larger than the largest distance for which such a change is thought possible. It should be mentioned that retrograde orbits are as renowned for their non-resonant stability as the prograde are for their unstable behaviour. Based on a large number of simulations of probes in different orbits around a selection of Gaussian shapes, we see that this property also holds for excited rotation of the central body. This property indicates that the resonances of these trajectories are narrow.

In Scheeres et al. (1996), a similar analysis like the one above has been presented where the Jacobi integral's consequences were studied. In addition to representing solutions for general inclinations implied by such a conserved quantity, we have here also explicitly determined their regions of applicability. If we for the moment restrict our attention to uniaxial rotation, Scheeres et al. (1996) used the Jacobi integral to determine whether or not an orbiting particle could have emanated from the central body. That is, if the particle's $\chi$ is smaller than the value of the effective potential $U=V+W$, Eq. (14), evaluated at the central body's surface, it follows that $\dot{\boldsymbol{r}}_{\mathrm{b}}^{2}$ for the particle is negative there.

A graphical presentation of this is included as Fig. 11, a plot of $U(r)$, for simplicity set to $U=V_{0}+W$, in the spin plane for our nominal nucleus parameters. The function peaks at the synchronous orbit

$r_{\mathrm{s}}=\left(\frac{\mu_{\mathrm{c}}}{\omega_{Z}^{2}}\right)^{1 / 3}$

which, unless the central body has some cohesion, is always larger than the largest physical dimension of the body in the spin plane, for convenience denoted $r_{\mathrm{c}}$. In such a circumstance, $\chi\left(r, \dot{\boldsymbol{r}}_{\mathrm{b}}=\mathbf{0}\right)=U(r)=U\left(r_{\mathrm{c}}\right)$ has two $r$ solutions, one at $r_{\mathrm{c}}$, and another one a distance beyond $r_{\mathrm{s}}$. Clearly, there exist a series of orbits with $U\left(r_{\mathrm{s}}\right)>\chi\left(r>r_{\mathrm{s}}\right)>U\left(r_{\mathrm{c}}\right)$ which do not originate from the central body either, and the criterion of Scheeres et al. (1996) is therefore, in a way, too strict. This nuance is however accounted for by Eqs. (57) and (59) which in the spin plane reduces to what we have discussed above.

In general, particles in a prograde orbit do not come from, or an orbiter does not collide with the central body if Eq. (59) is

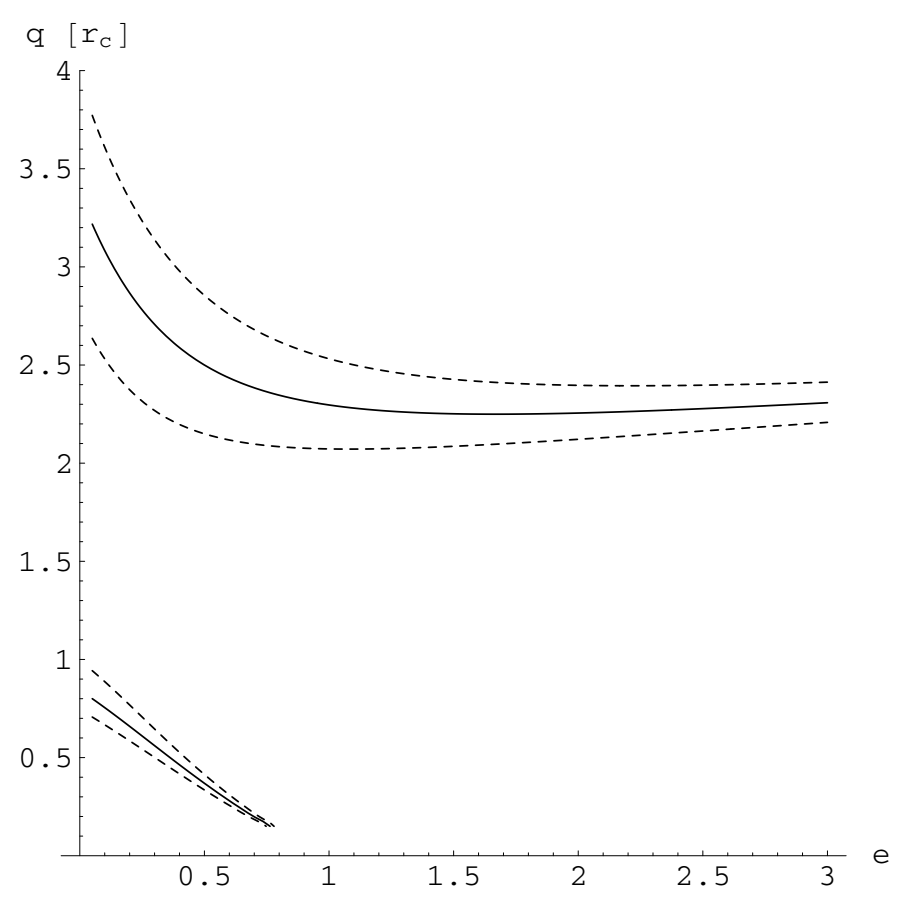

Fig. 12. Limiting contour (solid) for an $I_{0}=0, e_{0}=0.5, q_{0}=2.5 r_{\mathrm{c}}$ orbit in the nucleus spin plane, $I=0$. The dashed curves represent the displaced limiting contours if $\Omega$ is increased or decreased slightly.

false, $|\chi|>\left|U\left(r_{\mathrm{s}}\right)\right|$ in the spin plane for one-axis rotation, and if $q_{0}>q_{I=0}^{*}>r_{\mathrm{c}}$, corresponding to $r_{0}>r_{\mathrm{s}}>r_{\mathrm{c}}$ in the spin plane for the restricted problem. The lower left $e_{I=0}^{+}$branch of Fig. 9 represents the pocket of bound trajectories with $|\chi|>\left|U\left(r_{\mathrm{s}}\right)\right|$ and $r_{0}<r_{\mathrm{s}}$, see Fig. 11.

\section{Non-gravitational and tidal forces}

Shortly after comet rendezvous, the small module Philae will be separated from Rosetta so that it can land on 67P's nucleus. Although close prograde orbits undergo large and rapid changes in their parameters, they are attractive (Bertrand et al. 2004) for this particular phase; the relative speed between the lander and the nucleus is minimized so that Philae is not, for instance, buried beneath the comet surface (Kührt et al. 1997). This is made even more relevant by the fact that the lander was constructed for the probably lighter 46P/Wirtanen, as can be inferred from a comparison of the comet's perihelion water production rate and observed non-gravitational displacements of its orbit.

We have argued that, as long as certain precautions are met, Rosetta is unlikely to collide with the nucleus under the action of gravitation alone during such a maneuver. However, also to answer issues of impact avoidance on time-scales which stretch over several orbits, the rate at which solar radiation and outgassing pressure changes $\Omega$ must be addressed. The situation here is quite different from NEAR's retrograde landing on Eros which is almost thousand times more massive (Miller et al. 2002) than our nominal comet nucleus.

Before proceeding, we include a plot which illustrates how sensitive the minimum pericenter distance is for variations in $\Omega$. The situation in Fig. 12 is identical to one of the two orbits plotted in Fig. 9. This time however, the limiting contours for the cases where $\Omega_{0}$ of Eq. (48) is changed with $\Delta \Omega_{0}= \pm 0.05 \Omega$, 
with $\Omega$ equal to the value at the solid curve, are also added. We see that small variations in the modified Jacobi function induce fairly small relative variations in minimum $q$. A more convenient expression for the corresponding change in pericenter distance can be conjured by differentiating Eq. (61), resulting in

$$
\frac{\Delta q_{h}}{q_{h}}=2 \frac{\Delta \Omega}{\Omega} .
$$

\subsection{Outgassing pressure}

At the time when the lander is delivered, somewhere beyond the comet heliocentric distance of $R=3-4 \mathrm{AU}$, the sublimation of water from the nucleus is negligible (Crifo et al. 1999), but outgassing of more volatile species like $\mathrm{CO}$, less modulated by $R$ (Capria et al. 2004), could be significant.

With $\boldsymbol{f}$ as the perturbing force per mass unit, it follows from Eqs. (9) and (13) that the Jacobi function's associated rate of change obeys

$\dot{\chi}=\dot{\Omega}=\boldsymbol{f} \cdot \dot{r}_{\mathrm{b}}$.

Following Montenbruck \& Gill (2000), the pressure force on the orbiting spacecraft per mass unit exerted by the outflowing molecules of the coma is $\boldsymbol{f}_{\mathrm{c}}=A_{\mathrm{S} / \mathrm{C}} \rho v \boldsymbol{v} / m_{\mathrm{S} / \mathrm{C}}$ (the drag coefficient $C_{\mathrm{D}}$ is here set to two), where $\rho$ is the mass density of the coma, $v$ the relative velocity between probe and flow, and at last $m_{\mathrm{S} / \mathrm{C}}$ and $A_{\mathrm{S} / \mathrm{C}}$ are the spacecraft's mass and its gas exposed area, respectively. If not very close to the nucleus, where the flow could be almost along the comet surface (Crifo et al. $1995), \boldsymbol{v} / v=\boldsymbol{r} / r$ is a common assumption. This is related to the fact that while the cometocentric velocity of a probe in a bound orbit is $\sim 0.1 \mathrm{~m} \mathrm{~s}^{-1}$, the velocity of the collisionally accelerated outflowing molecules could typically be as high as $\sim 1 \mathrm{~km} \mathrm{~s}^{-1}$ (Crifo \& Rodionov 1997).

Writing $\boldsymbol{f}_{\mathrm{c}}=\Lambda(\boldsymbol{r}) \boldsymbol{r} / \mathrm{r}^{3}$, it has been shown, based on simulations of some cometary comae (Scheeres et al. 2000), that $\Lambda$ is approximately dependent on direction only, and not on distance. That is, if $\phi$ and $\theta$ are cometocentric direction angles, $\Lambda(r, \phi, \theta) \approx \Lambda(\phi, \theta)$. In particular, this dependency reappears in the simulations of 67P/Churyumov-Gerasimenko's coma by Crifo et al. (2004) in the solar direction where the pressure is strongest. A CO rate of $10^{27} \mathrm{~mol} . \mathrm{s}^{-1}$, a worst-case scenario (Bockelée-Morvan et al. 2004), seems (Crifo et al. 2004) to yield an approximate maximum $\Lambda_{\mathrm{M}}=0.1 r_{\mathrm{c}}^{3} \mathrm{~h}^{-2}$ for full exposure of the solar cell arrays. $r_{\mathrm{c}}$ here has nothing to do with the nucleus directly, but is just our prefered unit of length.

The outgassing induced change of $\Omega$ on an inbound leg from apocenter $p$ at time $t_{p}$, to pericenter $q$ at time $t_{q}$, can then easily be evaluated

$$
\Delta \Omega_{\mathrm{c}}=\int_{t_{p}}^{t_{q}} \mathrm{~d} t \frac{\Lambda}{r^{3}} \boldsymbol{r} \cdot \dot{\boldsymbol{r}}_{\mathrm{b}}=\int_{t_{q}}^{t_{p}} \mathrm{~d} t \Lambda \frac{\mathrm{d}}{\mathrm{d} t} \frac{1}{r}=\bar{\Lambda}\left(\frac{1}{p}-\frac{1}{q}\right)
$$

which amounts to only a few percent of $\Omega_{0}$ even if the angular mean $\bar{\Lambda}$ is set to $\Lambda_{\mathrm{M}}$, and the pericenter distance is a very close $q=2 r_{\mathrm{c}}$.

More interestingly, $\Omega$ decreases on the probe's journey towards the nucleus. And since impact avoidance of a probe in a prograde orbit is achieved by minimizing $\Omega$, i.e. maximizing its absolute value, Eq. (59), outgassing will actually make such an orbit safer from a dynamical point of view. For a retrograde orbit, the opposite occurs because large positive $\Omega$ 's are more favourable, Eq. (61), making an inbound orbit more hazardous under the influence of outgassing pressure.

\subsection{Solar radiation pressure}

If all incident photons are absorbed by Rosetta's large solar cell arrays, composed of specially developed non-reflective sillicon cells, the induced force per mass unit acting on the spacecraft can be written

$\boldsymbol{f}_{\odot}=-\frac{\Theta}{R^{2}} \boldsymbol{u}_{\odot}, \quad \Theta=0.001 r_{\mathrm{c}} h^{-2}$.

$R$ is as before the comet's heliocentric distance and $\boldsymbol{u}_{\odot}$ a cometocentric unit vector in the direction of the Sun. $\Theta$ is a strength parameter equal to the given high value for maximum area exposure to solar radiation, and if $R$ is dimensionless and evaluated in astronomical units.

It is straightforward to show that the radiation pressure induced acceleration can be derived from a potential $V_{\odot}=-\boldsymbol{f}_{\odot} \cdot \boldsymbol{r}$, but the effect cannot be included by merely adding this potential to the Jacobi function. This is due to the potential's explicit dependency on time, see Eq. (13), which changes $\Omega$ at a relatively rapid pace. Therefore, a direct approach is more advisable. Using Eq. (65) we have

$\Delta \Omega_{\odot}=-\frac{\Theta}{R^{2}} \int_{t_{0}}^{t} \mathrm{~d} t\left[\boldsymbol{u}_{\odot} \cdot \dot{\boldsymbol{r}}-\boldsymbol{u}_{\odot} \cdot(\boldsymbol{\omega} \times \boldsymbol{r})\right]$,

where the second of the bracketed terms is of most interest since its maximum is dominant at distances above the synchronous radius $a=1.6 r_{\mathrm{c}}$ for the adopted rotation period, Table 2.

On one leg of an orbit, the radiation pressure induced change of the Jacobi function is hence approximately bounded by

$\left|\Delta \Omega_{\odot}\right|<\frac{\Theta}{R^{2}} \frac{\omega_{Z}}{G} \int_{-\pi}^{0} \mathrm{~d} f r^{3}=\frac{\Theta}{R^{2}} \frac{\omega_{Z}}{\sqrt{\mu_{\mathrm{c}}}} a^{5 / 2} \pi\left(1+\frac{1}{2} e^{2}\right)$

with the use of the relation $\mathrm{d} t=\mathrm{d} f r^{2} / G$. The relative alteration is

$\left|\frac{\Delta \Omega_{\odot}}{\Omega}\right|<\frac{\Theta}{R^{2}} \frac{\pi}{\mu_{\mathrm{c}}} \frac{1+1 / 2 e^{2}}{\sqrt{1-e^{2}}} a^{2}$

which again amounts to only a few percent for the probe in an eccentric $a=5 r_{\mathrm{c}}$ orbit at $R=3 \mathrm{AU}$. However, due to the quadratic semi-major axis dependency of Eq. (70), more distant orbits could accumulate non-negligible changes in $\Omega$. These can, on the other hand, be reduced with a proper choice of orbital geometry, minimizing the integrated part of Eq. (68). If the solar cell arrays do not rotate relative to the direction of the Sun, the secular problem is solvable (Mignard \& Henon 1984) in terms of the heliocentric distance $R$, i.e. the induced changes of the orbit are slow. 


\subsection{Tidal acceleration}

An orbiting spacecraft is also affected by the gravitational pull from the Sun which, if described relative to the comet, is countered by an effective acceleration equal in magnitude to the heliocentric acceleration of the nucleus. Although the highly elliptic orbit of the nucleus does not make it clear that such concepts can be used, the effect of the solar tidal acceleration can nevertheless be gauged by calculating the "capture radius" of this isolated problem. The radius of the Hill sphere (Murray \& Dermott 1999) is

$r_{\mathrm{H}}=R\left(\frac{\mu_{\mathrm{c}}}{3 \mu_{\odot}}\right)^{1 / 3}=270 r_{\mathrm{c}}$

where the tabulated value is at comet heliocentric distance $R=$ $3 \mathrm{AU}$. At the same $R$, the solar radiation pressure acceleration equals the zero-order gravitational pull from the nucleus at $r=$ $100 r_{\mathrm{c}}$. Of course, solar radiation pressure will strip the probe from the comet below this distance. That is, tidal acceleration is negligible for the applications in this paper.

\section{Conclusions}

For a probe under the gravitational attraction of a uniformly rotating irregular central body, there exists an integral of motion called the Jacobi constant. If its implications are to be of relevance to the Rosetta mission a priori, one must investigate what happens to this function if the target comet's rotation is not uniaxial.

We have argued that there exists a Tisserand-like quantity, identical to the Jacobi constant for non-excited rotation, which is sufficiently conserved in general for it to have interesting implications for the evolution of the probe's orbit. In particular, its near constancy can be used to design close prograde orbits which under the action of gravitation alone do not evolve into collision trajectories with the comet nucleus. These orbits are relevant for the lander delivery phase of the Rosetta mission despite their notoriously unstable nature.

An analysis based solely on the Tisserand-like quantity's conservation shows that impact is best avoided if the probe's initial orbit is chosen to be retrograde or prograde with its initial cometocentric velocity lying in the plane normal to the nucleus spin. Non-gravitational forces are, for our chosen nominal nucleus at heliocentric distance 3-4 AU, not seen to change the pseudo-integral enough to affect the above conclusions in a worst-case scenario. More specifically, the adopted radial outgassing pressure makes the unstable close prograde orbits slightly safer, while close retrograde trajectories become somewhat more hazardous.

Acknowledgements. This work was done as a part of project 153382/V30, The Research Council of Norway.

\section{References}

Beletskii, V. V. 1966, Motion of an Artificial Satellite About Its Center of Mass, Mechanics of Space Flight Series, Israel Program for Scientific Translations, Jerusalem

Bertrand, R., Ceolin, T., \& Gaudon, P. 2004, Rosetta Lander Descending Phase on the Comet 67P/Churyumov-Gerasimenko, 18th International Symposium on Space Flight Mechanics

Bockelée-Morvan, D., Moreno, R., Biver, N., et al. 2004, in The New ROSETTA Targets, ed. L. Colangeli, E.M. Epifani, \& P. Palumbo, (Dordrecht: Kluwer), ASSL, 311, 25

Byrd, P. F., \& Friedman, M. D. 1971, Handbook of Elliptic Integrals for Engineers and Scientists (Berlin: Springer-Verlag)

Capria, M. T., Coradini, A., De Sanctis, M. C., et al. 2004, in The New ROSETTA Targets, ed. L. Colangeli, E. M. Epifani, \& P. Palumbo, (Dordrecht: Kluwer), ASSL, 311, 177

Chauvineau, B., Farinella, P., \& Mignard, F. 1993, Icarus, 105, 370

Crifo, J. F., \& Rodionov, A. V. 1997, Icarus, 127, 319

Crifo, J. F., Itkin, A. L., \& Rodionov, A. V. 1995, Icarus, 116, 77

Crifo, J. F., Rodionov, A. V., \& Bockelée-Morvan, D. 1999, Icarus, 138,85

Crifo, J. F., Lukyanov, G. A., Zakharov, V. V., et al. 2004, in The New ROSETTA Targets, ed. L. Colangeli, E. M. Epifani, \& P. Palumbo, (Dordrecht: Kluwer), ASSL, 311, 119,

Davidsson, B. J. R, \& Gutiérrez, P. J. 2005, Icarus, 176, 453

Garmier, R., \& Barriot, J.-P. 2001, Cel. Mech., 79, 235

Goldstein, H. 1980, Classical Mechanics, Addison-Wesley, Reading

Gutiérrez, P. J., Jorda, L., Samarasinha, N. H., et al. 2003, Outgassinginduced effects in the rotational state of comet $67 \mathrm{P} /$ ChuryumovGerasimenko during the Rosetta mission, DPS 35th Meeting

Heiskanen, W. A., \& Moritz, H. 1967, Physical Geodesy (San Francisco: W.H. Freeman and Company)

Hitzl, D. L., \& Breakwell, J. V. 1971, Cel. Mech., 3, 346

Kinoshita, H. 1972, PASJ, 24, 423

Kührt, E., Knollenberg, J., \& Keller, H. U. 1997, Planet. Space Sci., 45,665

Lamy, P. L., Toth, I., Weaver, H., et al. 2003, The Nucleus of Comet 67P/Churyumov-Gerasimenko, the New Target of the Rosetta Mission, DPS 35th Meeting

Lichtenberg, A. J., \& Lieberman, M. A. 1992, Regular and Chaotic Dynamics (New York: Springer-Verlag)

Mignard, F., \& Henon, M. 1984, Cel. Mech., 33, 239

Miller, J. K., Konopliv, A. S., Antreasian, P. G., et al. 2002, Icarus, 155,3

Montenbruck, O., \& Gill, E. 2000, Satellite Orbits (Germany: Springer-Verlag)

Muinonen, K. 1998, A\&A, 332, 1087

Muinonen, K., \& Lagerros, J. S. V. 1998, A\&A, 333, 753

Murray, C. D., \& Dermott, S. F. 1999, Solar System Dynamics (Cambridge: Cambridge University Press)

Mysen, E. 2004, Planet. Space Sci., 52, 897

Rickman, H., Kamél, L., Festou, M. C., et al. 1987, ESA SP-278, 471

Rossi, A., Marzari, F., \& Farinella, P. 1999, Earth, Planets Space, 51, 1173

Roy, A. E. 1998, Orbital Motion (Bristol: Institute of Physics Publishing)

Scheeres, D. J., Ostro, S. J., Hudson, R. S., et al. 1996, Icarus, 121, 67

Scheeres, D. J., Bhargava, S., \& Enzian, A. 2000, TMO Progress Rep., 42 